\title{
Matrix metalloproteinases in lung repair
}

\author{
W.C. Parks
}

The aims of this article are to discuss current concepts of the function of matrix metalloproteinases (MMPs), with an emphasis on identification of authentic substrates. Focusing on matrilysin (MMP-7), it is demonstrated how a single extracellular proteinase can regulate key and common functions of epithelial biology, namely defence against microorganisms, re-epithelialisation in response to injury and acute inflammation. Using findings from models of acute lung injury, the mechanism of matrilysin's action in these processes, that is, the proteins it acts upon to facilitate specific biological processes is discussed.

Despite their specialisation to serve distinct functions, the epithelium of different tissues respond similarly to injury and infection and regulate inflammation by equivalent mechanisms. By forming a barrier and releasing antimicrobial products, epithelia provide the first line of defence against invading pathogens. Following injury, epithelial cells initiate a programmed series of coordinated responses to restore tissue integrity. By their production of chemoattractants, adhesion molecules, and other proteins, epithelial cells recruit and confine inflammatory cells to sites of injury. Though seemingly divergent processes, the epithelial programmes regulating repair, defence and inflammation may have coevolved, particularly with respect to the genes selectively induced. After all, injury provides an opportunity for infection, which can lead to injury; both events being proinflammatory. Hence, many of the epithelial products associated with any one of these events are likely common to all. Matrilysin (MMP-7), is an example of a protein that functions in defence, repair, and as shown here, inflammation.

MMPs comprise a family of 23 (currently) related, yet distinct enzymes. MMPs are secreted or anchored to the cell surface $[1,2]$, thereby confining their catalytic activity to membrane proteins or proteins within the secretory pathway or extracellular space. As their name suggests, MMPs are thought to be responsible for the turnover and degradation of connective tissue proteins, a function that is likely performed by some family members. However, matrix degradation is neither the sole nor shared function of these enzymes. Several reports in recent years have demonstrated that various MMPs can activate a variety of nonmatrix proteins, including cytokines, chemokines, receptors and antimicrobial peptides [3]. Thus, MMPs should not be viewed solely as proteinases of matrix catalysis, but rather, as mentioned above, as extracellular processing enzymes involved in regulating cell-cell and cell-matrix signalling events.

The use of genetically defined mice has allowed investigators to uncover specific and, at times, unexpected functions of MMPs. Of the MMPs targeted to date, all, except for MT1MMP, show no or only a minor phenotype in unchallenged

Correspondence: W.C. Parks, Dept of Pediatrics, Washington University School of Medicine, Box 8208, 660 S. Euclid Avenue, St Louis, MO 63110, USA. Fax: 1 3142862894. E-mail: parks_w@kids.wustl.edu mice, and few MMPs seem to have a direct role in turnover or degradation of extracellular matrix proteins [2, 3]. These observations indicate that many MMPs do not serve vital functions in development or homeostasis; many are not even expressed in the foetus. In contrast, once challenged, MMPknockout mice reveal a variety of phenotypes indicating that these enzymes serve specific, and at times essential roles in tissue repair, angiogenesis, host defence, inflammation and tumour progression, among other processes. Thus, it seems that several MMPs, at least most of those that have been knocked out, have evolved to respond to the insults and pressures of extra-uterine existence.

Matrilysin is produced by the epithelium of several noninjured, noninflamed tissues, such as lung, liver and breast. The expression of matrilysin in healthy epithelium suggests that it functions in a common homeostatic process among epithelia, which seems to be defence against microorganisms. In mice, matrilysin activates intestinal pro- $\alpha-$ defensins (cryptdins), a family of antimicrobial peptides, and due to the lack of mature $\alpha$-defensins, matrilysin-null (MAT-/-) mice have an impaired ability to battle enteric pathogens [4]. In addition to being produced in intact tissues, matrilysin is expressed in migrating epithelium in injured airway and intestine, suggesting that this MMP also functions in epithelial repair. Indeed, in wildtype (WT) tissue, trachea wounds repair rapidly, whereas wounds in MAT-/- tissue show no evidence of epithelial migration, even several days post-injury [5]. In fact, MAT-/- mice have the most severe wound repair defect among the MMP-knockout mice generated to date [2].

As matrilysin is needed for the closure of mucosal wounds, it was reasoned that MAT-/- mice would be more susceptible to severe tissue injury. After all, if re-epithelialisation is impaired, then excessive fibrosis due to continued exudation of interstitial fluids, granulation tissue formation and fibroblast migration, would ensue. This idea was tested using the bleomycin-induced model of lung fibrosis. Bleomycin, instilled intratracheally, causes rapid and extensive alveolar epithelia damage, followed by severe, persistent inflammation and fibrosis

Matrilysin was not expressed in control mouse lungs but was induced in alveolar epithelial cells bordering denuded regions following instillation with 0.04 units of bleomycin (fig. 1a). Contrary to expectations, both the protein levels in bronchoalveolar lavage (BAL) and the deposition of new collagen were slightly less in MAT-/- mice compared with WT mice. To explore further this unexpected response, mice were instilled with $0.02-16$ units of bleomycin and their survival tracked over the next 30 days (fig. 1b). Quite surprisingly, MAT-/- mice were protected against bleomycin-induced lethality. With high doses of bleomycin, most WT mice were dead by 10 days after instillation, while most MAT-/mice were still alive (fig. $1 \mathrm{c}$ and $\mathrm{d}$ ).

The slight reduction in fibrosis was not thought to have led to protection against bleomycin-induced lethality. Rather, it was reasoned that matrilysin regulates a potentially lethal process during the acute phase of injury, and this process 
was determined as the transepithelial influx of neutrophils. Whereas several neutrophils were detected in BAL from WT mice, few were recovered from the BAL of MAT-/- mice. However, the total neutrophils isolated from bleomycininstilled lung tissue did not differ between WT and MAT-/mice. Thus, although approximately the same number of neutrophils emigrated into MAT-/- and WT lungs soon after injury, these cells had an impaired ability to advance from the interstitium into the lumenal compartment in the absence of matrilysin. Indeed, immunostaining for neutrophils revealed that these granulocytes were stuck within the alveolar wall or perivascular compartments (fig. 2). Since matrilysin is not expressed by murine neutrophils, endothelial or interstitial cells, the defect in neutrophil migration must be due to an epithelial-derived process.

Based on these findings, it was reasoned that matrilysin regulates the activity of an epithelial-derived neutrophil chemotactic factor. The levels of $\mathrm{KC}$, a murine $\mathrm{CXC}$ chemokine, a functional homologue of interleukin (IL)-8 and a structural homologue of growth-related protein- $\alpha$ were assessed. $\mathrm{KC}$ is a potent and essential neutrophil chemoattractant and is rapidly expressed by epithelial, endothelial, and interstitial cells in a variety of inflammatory conditions, including lung injury and infection.

$\mathrm{KC}$ levels in BAL from WT lungs were elevated within $12 \mathrm{~h}$ post-bleomycin and returned to controls levels by 3 days. In contrast, $\mathrm{KC}$ protein remained at or near to baseline levels in BAL from bleomycin-treated MAT-/- mice. Based on equivalent messenger ribonucleic acid levels, reduced $\mathrm{KC}$

a)
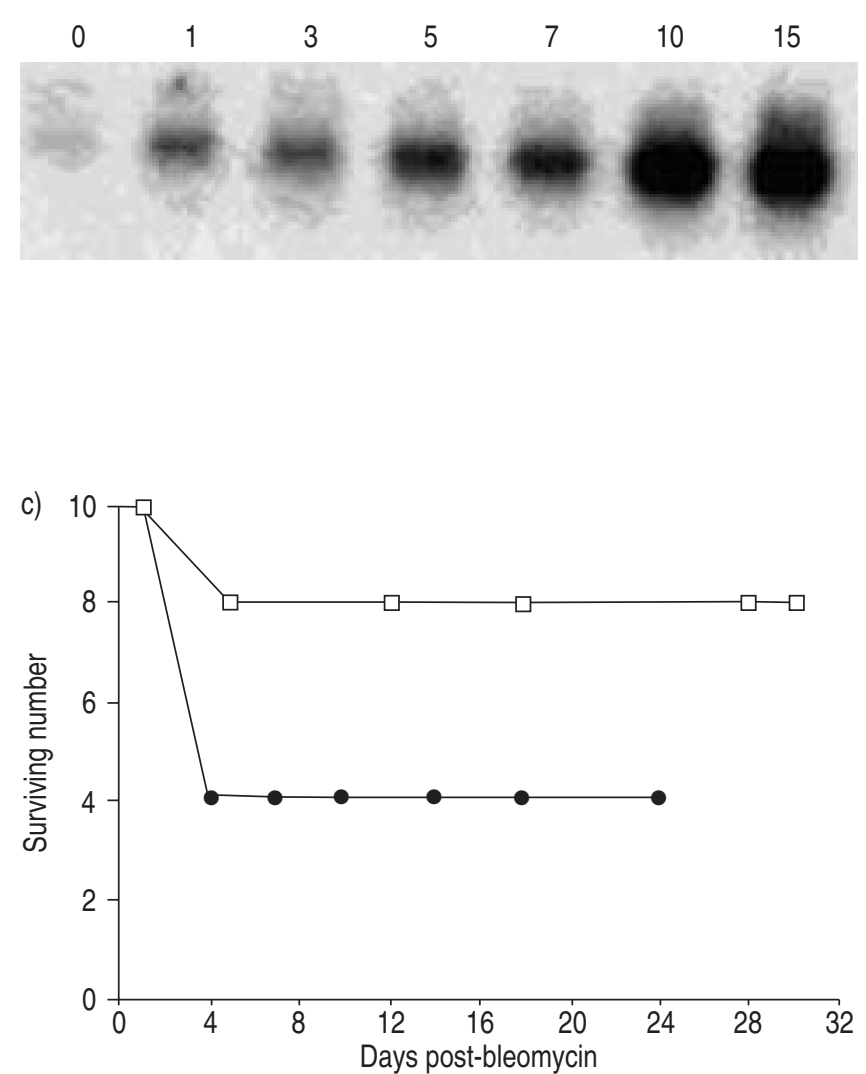

protein in the BAL of MAT-/- mice was not due to decreased expression, and elevated levels in the serum indicated that endothelial-derived $\mathrm{KC}$ was essentially equal in both mouse strains. Of significance, whereas $\mathrm{KC}$ protein levels remained fairly constant in lung extracts from WT mice, they accumulated markedly in lungs of MAT-/- mice.

These data were interpreted to indicate that matrilysin acts on a protein that facilitates the distribution of $\mathrm{KC}$ from the interstitium to the lumen, and the author's group focused on syndecan-1. IL-8, the human homologue of $\mathrm{KC}$, requires heparan sulphate to attract neutrophils and to bind its receptor, CXCR2, with high affinity. Syndecans, a family of four transmembrane proteins, are the principal source of cell-surface heparan sulphate and can interact via their carbohydrate chains with a variety of proteins, including chemokines [6]. Syndecans are shed in response to injury and inflammation, and this process is thought to be required for their biological activity. Since syndecan-1 is abundantly expressed on the basal surface of lung epithelial cells, it was determined whether it is the matrilysin substrate involved in mobilisation of $\mathrm{KC}$.

In untreated WT and MAT-/- mice, an equivalent signal for syndecan-1 was seen in the conducting airways and alveoli, and similar levels of the proteoglycan were detected in detergent extracts of whole lung. In bleomycin-treated WT lungs, extensive areas of alveoli lacking staining for syndecan-1, a slightly reduced signal in conducting airways and a marked increase in soluble syndecan-1 in BAL were found. In MAT-/- mice, however, both the pattern and

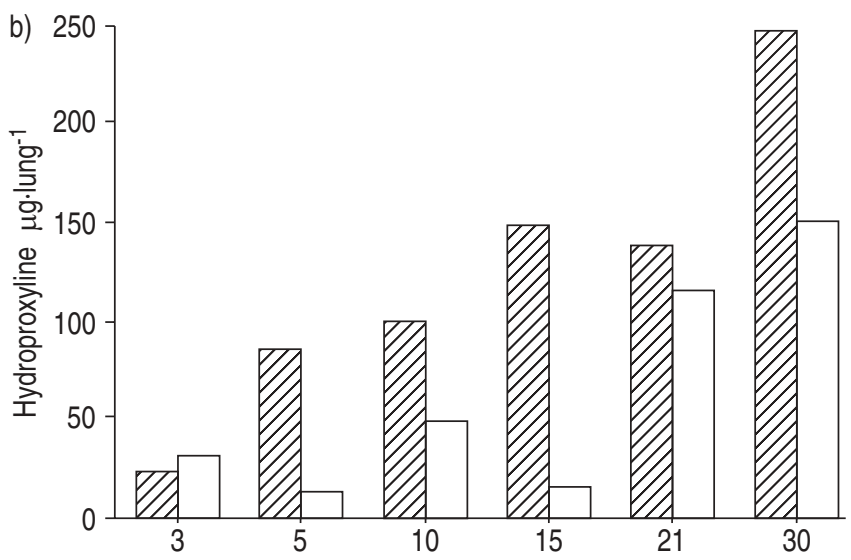

d)

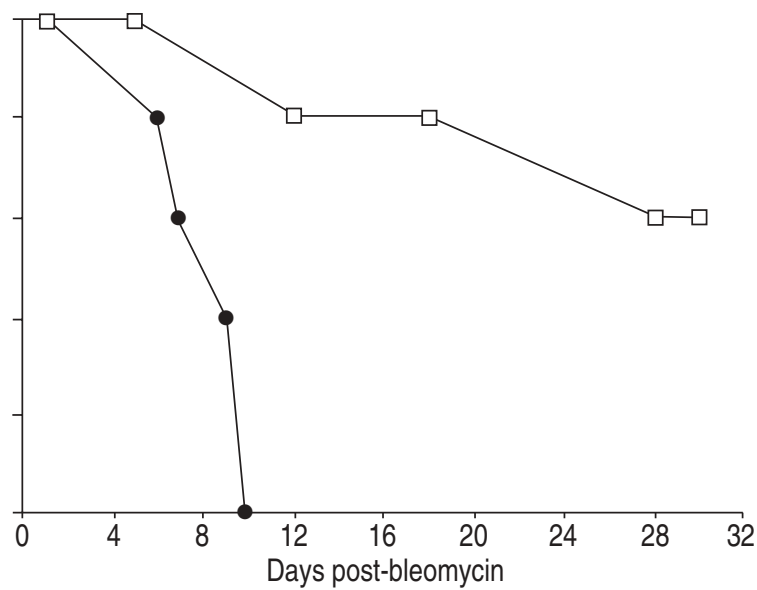

Fig. 1.-Reduced fibrosis and enhanced survival in matrilysin-null mice. a) Matrilysin expression was assessed by reverse-transcriptase polymerase chain reaction/Southern hybridisation at various days post-instillation of $0.04 \mathrm{U}$ bleomycin. b) Total collagen was assessed by hydroxyproline levels in total lungs from wildtype $(\mathbb{Z})$ and matrilysin-null $(\square)$ mice instilled with $0.04 \mathrm{U}$ bleomycin. Wildtype $(\bullet)$ and matrilysin-null $(\square)$ were instilled with c) $0.04 \mathrm{U}$ and d) $0.16 \mathrm{U}$ bleomycin, and survival was followed for 30 days. 


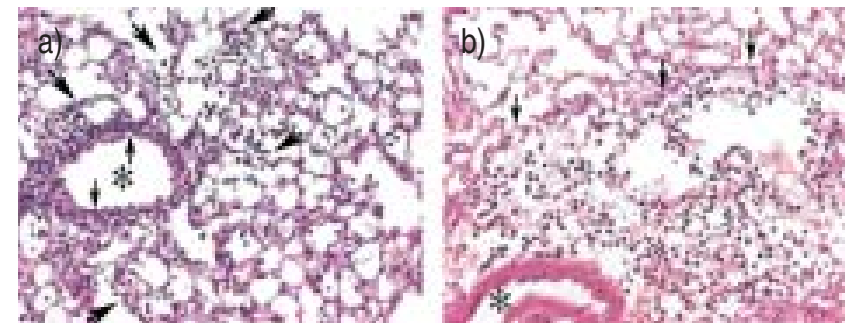

Fig. 2.-Impaired neutrophil migration in matrilysin-null mice. The lumen of a venule is marked by an asterisk, the border between the perivascular and alveolar compartments is marked by the small arrows, and the neutrophils that have migrated into the alveoli are indicated by the large arrows.

intensity of syndecan-1 staining did not change compared with that seen in control lungs and soluble syndecan-1 was not detected in BAL samples. Using immunoprecipitation approaches, $\mathrm{KC}$ was demonstrated to be bound to shed syndecan-1. Furthermore, as in MAT-/- mice, KC levels and neutrophil numbers were reduced in BAL from syndecan-1null mice compared with WT mice. In the absence of syndecan-1 or matrilysin, $\mathrm{KC}$ is not translocated into the alveolar space and accumulates in the tissue space. Together, these data indicate that $\mathrm{KC}$ is bound to matrilysin-shed syndecan- 1 and that this association is needed to shuttle $\mathrm{KC}$ into the injured alveolar space (fig. 3).

\section{Conclusion}

These data describe a mechanism in which three epithelial components, a secreted proteinase (matrilysin), a cell-bound proteoglycan (syndecan-1), and a chemokine (KC), act coordinately to control and confine inflammation to sites of injury $[7,8]$. The author's group propose that in response to injury, epithelial cells synthesise, secrete and deposit KC (or IL-8 in humans) onto the pre-existing syndecan-1 molecules on their basal surface. Matrilysin is also induced by injury and cleaves the syndecan-1 core protein to release the ectodomain-KC complex. The shed complex is then transported, either actively or passively, to the apical surface

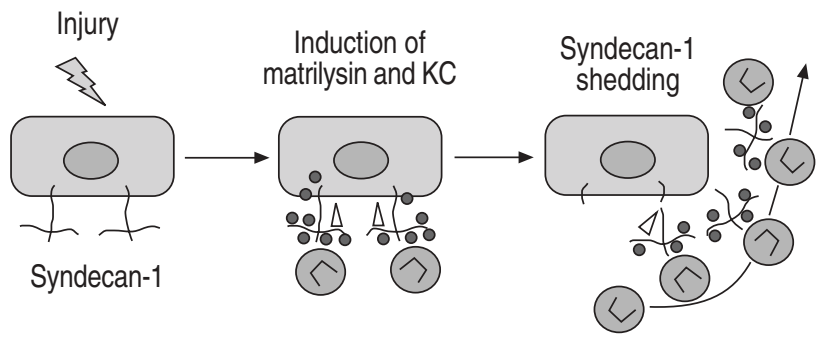

Fig. 3. - Matrilysin shedding of syndecan-1 establishes a chemokine chemotactic gradient that guides the transepithelial influx of neutrophils at sites of injury. $\bullet$ : KC; $\triangle$ : matrilysin.

creating a chemotactic gradient guiding neutrophils into the alveolar space.

\section{References}

1. $\mathrm{Vu} \mathrm{TH}$, Werb Z. Matrix metalloproteinases: effectors of development and normal physiology. Genes Dev 2000; 14: 2123-2133.

2. Parks WC, Shapiro S. Matrix metalloproteinases in lung biology. Respir Res 2001; 2: 10-19.

3. McCawley LJ, Matrisian LM. Matrix metalloproteinases: they're not just for matrix anymore!. Curr Opin Cell Biol 2001; 13: 534-540.

4. Wilson CL, Ouellette AJ, Satchell DP, et al. Regulation of intestinal a-defensin activation by the metalloproteinase matrilysin in innate host defense. Science 1999; 286: 113-117.

5. Dunsmore SE, Saarialho-Kere UK, Roby JD, et al. Matrilysin expression and function in airway epithelium. $J$ Clin Invest 1998; 102: 1321-1331.

6. Park PW, Reizes O, Bernfield M. Cell surface heparan sulfate proteoglycans: selective regulators of ligand-receptor encounters. J Biol Chem 2000; 275: 29923-29926.

7. Li Q, Park PW, Wilson CL, Parks WC. Matrilysin shedding of syndecan-1 regulates chemokine mobilization and transepithelial efflux of neutrophils in acute lung injury. Cell 2002; 111: 635-646.

8. Shapiro SD. Immunology: Mobilizing the army. Nature 2003; 421: 223-224. 